
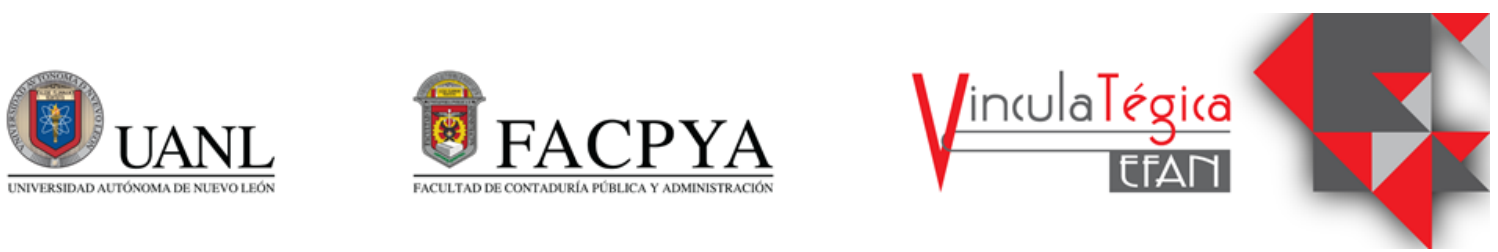

\title{
Utilización de encuesta de escala Likert para precisar el grado de aplicación del modelo de control interno COSO 2013
}

\author{
Juan Lorenzo Barrera Guerra Jr. ${ }^{1}$ y Verónica Hinojosa Cruz Adriana ${ }^{2}$ \\ ${ }^{I}$ Universidad Autónoma de Nuevo León, Facultad de Contaduría Pública y Administración Monterrey, Nuevo \\ León, México, juan_lorenzo79@hotmail.com,Av.Universidad S/N \\ Col. Ciudad Universitaria, (+52) 8180226797 \\ ${ }^{2}$ Universidad Autónoma de Nuevo León, Facultad de Contaduría Pública y Administración Monterrey, Nuevo \\ León, México,avhinojosacruz@gmail.com,Av.Universidad S/N \\ Col. Ciudad Universitaria, (+52) 8182803799
}

Información del artículo revisado por pares
Fecha de aceptación: junio-2021
Fecha de publicación en linea: diciembre-2021
DOI: https://doi.org/10.29105/vtga7.2-47

\section{Resumen}

En la investigación científica pudiera ser prevaleciente la existencia de variables cualitativas que no cuenten con información institucional que permita precisar su nivel de aplicación o estado actual, como el caso de la implementación del Modelo de Control Interno COSO 2013 en las empresas listadas en los mercados financieros de la bolsa mexicanas de valores que no son requeridas para presentar información al respecto de forma institucional, al no existir una regulación que así lo demande, para dichos casos pudiera ser de utilidad la aplicación de un instrumento de medición de este tipo de variables como la encuesta de escala de tipo Likert definida con las bases teóricas del objeto en estudio, realizando los análisis de confiabilidad que requiere la encuesta previo a su aplicación y el establecimiento de los ítems apropiados para su desarrollo, esto anticipadamente a aplicar la misma a la población de estudio, para de esta forma poder precisar el estado actual de la variable.

Palabras Clave: Control Interno, COSO y Escalas Likert

Código JEL: M40

\begin{abstract}
Difficulty to find official information about qualitative variables on scientific research, for proving level or current status of them, could be an ordinary issue, such as case of the publicly listed companies in the Mexican Stock Exchange (BMV), in which the internal control framework applied by firms here listed is not a requirement of local Laws, therefore it could be a problem to find in companies reports, levels of COSO 2013 (Internal Control Framework) rollout. Likert Scale Survey could be a convenient instrument to solve this kind of issues, in this case survey must be designed using basics of COSO 2013 framework, evaluating contents reliability of it and also determining appropriated items for final document, these steps are highly recommended to ensure reliability of instrument.
\end{abstract}

Keywords: Internal Control, COSO \& Liker Scale. JEL: M40. 


\section{INTRODUCCIÓN}

Los escándalos financieros de los años 2001 y 2002 en Estados Unidos generaron desconfianza en el público inversionista de las bolsas de valores de dicho país, lo que cambio la visión de lo que se necesitaba de las empresas emisoras de valores (Empresas Públicas) en el mercado bursátil americano, que era regulado por la Securities Exchange Commision (SEC), dicho organismo tiene como misión la protección de los fondos de los inversionistas en la bolsa de valores en Estados Unidos, el mantener la operación de los mercados de valores ordenada y eficiente, así como facilitar la formación de capital, (Buzo, 2014).

Esto provocó que en Julio de 2002 el presidente George W. Bush promulgará en Estados Unidos la Ley Sarbanes Oxley, que fungía como la Ley de Mercado de Valores en dicho país, la cual ordenaba una serie de reformas para optimizar la responsabilidad corporativa, mejorar las divulgaciones financieras y combatir el fraude corporativo y contable, creando la Public Company Accounting Oversight Board (PCAOB), la cual estaría enfocada en la supervisión de las actividades de los contadores públicos independientes. (Arwinge, 2013).

Adicionalmente, en el caso de Estados Unidos, las empresas públicas tienen la obligación de aplicar un Marco de Control Interno, el cual se ha confirmado que el emitido por COSO es el más utilizado por estas empresas (Hirt, 2015).

Es importante puntualizar que en México no existe un indicador o regulación que requiera a las empresas públicas la aplicación de un Modelo de Control Interno especifico, similar al caso de Estados Unidos, lo cual se vuelve un área de oportunidad en el ámbito de la investigación científica.

Debido a esta problemática solo se tendría claridad del Modelo de Control Interno aplicado en las empresas públicas mexicanas que adicionalmente esta listadas en Estados
Unidos, al publicar esto en los informes anuales presentados ante la SEC. Bajo esta premisa, resulta de utilidad conocer el grado de aplicación del Modelo de Control Interno COSO 2013 en las empresas públicas en México aún en aquellas que no se encuentren listadas en Estados Unidos de Norteamérica.

Para tal efecto, en la ponencia, se presenta el objetivo de la misma, después se conceptualiza el Modelo COSO, así como la encuesta Likert como un instrumento de recolección de datos de percepción. Asimismo, se describen los elementos a cubrir en la construcción de la misma integrando de manera general, los principios de COSO. Al final, se concluye sobre su pertinencia.

\section{OBJETIVO}

Esta investigación de tipo descriptivo pretende fundamentar a través de las bases teóricas aplicables la utilización de una escala Likert para precisar el grado de implementación del Modelo de Control Interno COSO 2013 en las empresas públicas mexicanas que no cotizan en los mercados financieros en Estados Unidos, debido a que no existen datos formales en dichas compañías respecto a esto.

\section{MARCO TEÓRICO}

En este apartado, se fundamentan los antecedentes recientes del control interno, la regulación mexicana y por supuesto, la encuesta Likert y sus elementos.

\subsection{Ley Sarbanes Oxley y aplicación del Modelo de Control Interno COSO 2013}

Los Control Internos sobre la Información Financiera (ICFR) tomaron relevancia en el 2002 cuando el Congreso de Estados Unidos aprobó la Ley Sarbanes-Oxley (Ley de Mercado de Valores), que establecía las disposiciones relacionadas con la sección 404 de dicha Ley, instituyendo que:

a) La SEC prescribirá las reglas requeridas en la sección 13 (a) o 15 (d) de la Ley de Mercado de Valores de 1934 (15 U.S.C. $78 \mathrm{~m}$ o 780 (d)) requiriendo ahora que 


\section{los reportes anuales incluyan un informe de control interno, en el que se:}

(1) Precise la responsabilidad de la administración para establecer y mantener una estructura adecuada de ICFR.

(2) Así también que contenga una evaluación, sobre la efectividad de la estructura del ICFR por parte de la firma contadores públicos independientes que emite los informes financieros del emisor, esto al final del año fiscal.

b) Evaluación e Informe de Control Interno. - Con respeto a la evaluación de control interno requerida en la sección anterior, las firmas de contadores públicos que preparen o emitan informes de auditoría deberán dar fe e informar sobre la evaluación realizada por la administración de la empresa pública, (Sarbanes-Oxley Act, 2002).

De acuerdo con lo mencionado, las empresas listadas en los mercados financieros de Estados Unidos presentan dentro de sus informes anuales el resultados de la Evaluación del ICFR realizado por la administración, así como la opinión del Contador Público Independiente respecto a la efectividad del ICFR; dentro de ambos informes se precisa el Modelo de Control Interno que fue utilizado por las firmas; en una consulta realizada a Paul Sobel, actual presidente de la organización COSO, dio referencia que el marco de Control Interno de su organización es el más utilizado por las compañías públicas en Estados Unidos.

El Marco de Control Interno COSO 2013 fundamenta las bases que las organizaciones deben seguir para la implementación de un sistema de control interno y poder lograr sus objetivos de Negocio: Operativos, de Información y de Cumplimiento; a través de la implementación de sus 5 componentes: Entorno de Control, Evaluación de Riesgos, Actividades de Control, Información y Comunicación y Supervisión y sus 17 principios, (Commitee of Sponsoring Organizations of the Treadway Commission, 2013). La aplicación de dicho modelo en las organizaciones pudiera promover el orden en las medidas para lograr los objetivos de negocio, ya que establecen fundamentos que las firmas deben seguir para la implementación de un sistema de Control Interno, que empuje al cumplimiento de los metas de Negocio.

Como parte del estudio se precisó que al cierre del ejercicio de 2019, existieron 15 organizaciones mexicanas que son Empresas Públicas en Estados Unidos, de las cuales 13 cotizan en los mercados accionarios de la Bolsa Mexicana de Valores mismas que son precisadas en la tabla 1.

El formato 20-F que funge como informe anual para empresas públicas en Estados Unidos que son foráneas, incluye la sección "Reporte Anual de la Administración sobre el ICFR" donde la gerencia de las emisoras tienen la obligación de revelar el Modelo de Control Interno que fue utilizados para evaluar la efectividad del ICFR, así como cualquier debilidad relevante en el ICFR, (United States Securities and Exchange, 2021), por tanto la información en esta población revelaría la aplicación o no del Modelo de Control Interno COSO 2013 en estas organizaciones, así como la efectividad del ICFR.

Se realizó un análisis para precisar este dato confirmando que las 13 empresas mexicanas públicas en ambos países se encuentran apegadas al Modelo de Control Interno COSO 2013 y la administración y el contador público independiente corroboraron la efectividad del ICFR, lo cual en dichos casos pudiera facilitar la definición del Modelo de Control Interno aplicado y sus efectos en las variables de generación de valor en las organizaciones como el caso de Rentabilidad y Productividad, así también lo útil que sería que en México existiera una regulación que empujará a las organizaciones a revelar y evaluar el sistema de Control Interno aplicado, el análisis se detalla en la figura siguiente. 


\begin{tabular}{|c|c|c|c|c|c|c|}
\hline \multirow[b]{2}{*}{ Emisora } & \multirow[b]{2}{*}{$\begin{array}{c}\text { Listada en } \\
\text { Mercado } \\
\text { Accionario - } \\
\text { BMV }\end{array}$} & \multirow[b]{2}{*}{$\begin{array}{c}\text { Año de ler } \\
\text { informe } \\
\text { anual ante } \\
\text { SEC }\end{array}$} & \multicolumn{4}{|c|}{ Evaluación de ICFR 2019} \\
\hline & & & $\begin{array}{c}\text { Utiliza COSO } \\
2013 \text { como ICFR }\end{array}$ & $\begin{array}{c}\text { Efectividad } \\
\text { ICFR según } \\
\text { Gerencia }\end{array}$ & $\begin{array}{c}\text { Opinión del } \\
\text { ICFR de Firma } \\
\text { Contable } \\
\text { Independiente }\end{array}$ & $\begin{array}{l}\text { Firma de } \\
\text { Auditoría }\end{array}$ \\
\hline 1. America Movil & $\checkmark$ & 2000 & $\checkmark$ & $\checkmark$ & $\checkmark$ & EY \\
\hline 2. Betterware de México & $\mathrm{X}$ & 2019 & En proceso & N/A & N/A & Deloitte \\
\hline 3. CEMEX & $\checkmark$ & 2004 & $\checkmark$ & $\checkmark$ & $\checkmark$ & KPMG \\
\hline 4. Coca-Cola FEMSA & $\checkmark$ & 2002 & $\checkmark$ & $\checkmark$ & $\checkmark$ & EY \\
\hline 5. Controladora Vuela Compañía de Aviación & $\checkmark$ & 2013 & $\checkmark$ & $\checkmark$ & $\checkmark$ & EY \\
\hline 6. Fomento Económico Mexicano & $\checkmark$ & 2002 & $\checkmark$ & $V$ & $\checkmark$ & EY \\
\hline 7. Grupo Aeroportuario del Centro Norte & $\checkmark$ & 2006 & $\checkmark$ & $V$ & $V$ & Deloitte \\
\hline 8. Grupo Aeroportuario del Pacifico & $\checkmark$ & 2005 & $\checkmark$ & $V$ & $V$ & KPMG \\
\hline 9. Grupo Aeroportuario del Sureste & $\checkmark$ & 2002 & $V$ & $V$ & $V$ & $\mathrm{PwC}$ \\
\hline 10. Grupo SIMEC & $\checkmark$ & 2002 & $\checkmark$ & $V$ & $\checkmark$ & Moore Global \\
\hline 11. Grupo Televisa & $\checkmark$ & 2002 & $V$ & $V$ & $\checkmark$ & KPMG \\
\hline 12. Industrias Bachoco & $\checkmark$ & 2003 & $\checkmark$ & $\checkmark$ & $\checkmark$ & Deloitte \\
\hline 13. Grupo TMM & $\checkmark$ & 2002 & $\checkmark$ & $V$ & N/A & $\begin{array}{c}\text { Grant } \\
\text { Thornton }\end{array}$ \\
\hline 14. Petróleos Mexicanos & $\mathrm{X}$ & 2000 & $\checkmark$ & $V$ & N/A & KPMG \\
\hline 15. Vista Oil \& Gas & $V$ & 2019 & No descrito & N/A & N/A & EY \\
\hline
\end{tabular}

Tabla 1 / Fuente: Elaboración propia, basado en información de la plataforma EDGAR de la Securities and Exchange Commission. 
La aplicación del Modelo de Control Interno de las empresas públicas mexicanas sin definición formal de este dato, al no existir una regulación que requiera a estas la revelación del Modelo de Control Interno aplicado, precisa fundamentos para analizar dicha variable a través de instrumentos de escalas de medición con especialistas de Control Interno en estas organizaciones.

\subsection{Regulación Mexicanas sobre el Control Interno}

En México no existe un indicador o regulación que requiera a las empresas listadas en los mercados de valores la aplicación de un Modelo de Control Interno especifico similar al caso de Estados Unidos.

Las Disposiciones de Carácter General Aplicables a las Emisoras de Valores y otros participantes del Mercado de Valores de México, establecen en el artículo 41 que las emisoras deberán revelar en su información trimestral y anual, las modificaciones que hubieren realizado a los lineamientos en materia de control interno y auditoría interna de la sociedad y de las personas morales que controlen, así como a las políticas y criterios contables de la sociedad, conforme a los cuales elaboraron sus estados financieros, (Secretaria de Gobernación, 2019), precisando que aunque se cuenta con esta regulación, no es requerido revelar el Modelo de Control Interno que es utilizado por las empresas emisoras.

Como referencia, los procesos de auditoría financiera conllevan la validación del sistema de Control Interno previo a la ejecución de la revisión para evaluar el riesgo de incorrección material en los Estados Financieros, es decir el auditor aplicará procedimientos de valoración del riesgo con el fin de disponer de una base para identificar y valorar los riesgos de incorrección material en los estados financieros y en las afirmaciones, (Auditing and Assurance Standards Board, 2009), aunque para el alcance de este trabajo no habría un efecto negativo.

Es importe indicar que las únicas empresas o grupos de empresas que están obligadas a dictaminarse para efectos financieros son aquellas que se encuentran en la Bolsa Mexicana de Valores, lo anterior encuentra su fundamento en el artículo 85 fracción III y artículo 87 fracción I de la Ley de Mercado de Valores (LMV), (Hernández Campos et al., 2019). Por lo que se pudiera precisar que al existir esta obligación los procesos de control interno en las empresas públicas mexicanas están siendo evaluados para los efectos mencionados, como lo requieren las normas internacionales de auditoría, sin embargo, no se tiene la certeza del Modelo de Control Interno aplicado.

Esto confirma que no existe información institucional requerida por las regulaciones mexicanas que permita corroborar el Modelo de Control Interno utilizado por las empresas públicas en México, que permitiría adicional a la identificación de dicho dato, medir también la efectividad del Modelo de Control Interno COSO 2013 en la generación de factores como productividad y rentabilidad.

\subsection{La Escala Likert para precisar el grado de aplicación de COSO 2013 en empresas públicas mexicanas, no listadas en Estados Unidos}

Dado las condiciones mencionadas para las empresas públicas mexicanas sin definición formal del Modelo de Control Interno aplicado, para precisar el nivel de implementación del Modelo de Control Interno COSO 2013 en esta población, es de ayuda utilizar un instrumento de medición de percepción, diseñado considerando las bases de los 5 componentes y 17 principios que establecer dicho modelo.

Las escalas de medición de categorías pudieran ser instrumentos útiles para medir de forma cuantitativa las variables cualitativas como las relacionadas con consideraciones, predilecciones, cualidades, de un sector determinado entre otros. Likert propone una técnica de elaboración de escalas que presenta un proceso de construcción más breve y sencillo que la técnica escalar Thurstone y que es tan fiable como esta, la técnica Likert aplica a Items dicotómicos como a los de muchos puntos, 
aunque se emplea con mucha mayor frecuencia en estos últimos, (Chica \& Castejón, 2006).

Son muchos los términos asociados a la escala de categorías como: escalas de clasificación, de juicio absoluto, cerrada, de valoración resumida, de múltiple elección, tipo Likert, entre otras. En cualquier caso, bajo todas estas denominaciones se hace referencia a un procedimiento de escalamiento en el que el sujeto asigna los estímulos a un conjunto específico de categorías o cuantificadores lingüísticos, en su mayoría, de frecuencia (siempre, a veces, nunca, etc,) o de cantidad (todo, algo, nada, etc.), (Osinski \& Bruno, 1998).

Bajo esta perspectiva se pudiera facilitar la medición los diferentes grados de aplicación del Modelo de Control Interno COSO 2013 en la población de estudio señalada, ya que a través de un instrumento de encuesta de Escala Likert diseñado con los bases del Modelo de Control Interno COSO, siendo este aplicado a los representantes de control interno o auditoría en la población a estudiar, se podría precisar el nivel de implementación de dicho Modelo en la población de estudio y a través de esta precisión analizar su correlación con las variable con bases cuantitativas como la eficiencia operativa y la rentabilidad.

Se ha precisado que la escala Likert maneja respuestas de tipo ordinal, así también que es el formato más habitual, confirmando que los procedimientos de categoría ordinal como este tienen la capacidad de estimar aproximadamente la intensidad de la impresión de la forma más sencilla posible para el encuestado y es difícilmente sustituible, (González Lorenzo et al., 1990).

Considerando esta definición con la variable a estudiar y la popularidad del instrumento de medición Escala Likert, se reafirma que dicho instrumento pudiera contar con cualidades requeridas para precisar el grado de aplicación del Modelo de Control Interno COSO 2013 en las empresas públicas mexicanas, sin definición formal de este dato.

\subsection{Encuesta-Escala Likert, diseño y} análisis de consistencia.

Como se mencionó, los cuestionarios basados en escalas de medición Likert han sido utilizados, en la comprobación de propiedades o cualidades relacionadas con opiniones, discernimientos o estimaciones.

Considerando la naturaleza de la variable aplicación de COSO 2013 en las empresas públicas mexicanas no listadas en los mercados de estados unidos al no existir datos métricos o cuantitativos de manera oficial para precisar su estado, es de utilidad la definición de un instrumento de medición ordinal como el caso de la escala Likert.

Namakforoosh indica que la construcción de Escalas Likert requiere 6 etapas:

1. Copilar ítems: que expresen un amplio rango de actitudes, desde extraordinariamente positivas hasta negativas como son:

1) Muy de acuerdo.

2) De acuerdo.

3) Ni de acuerdo ni en desacuerdo.

4) En desacuerdo.

5) Muy desacuerdo.

Cada ítem debe tener una puntuación, en este caso de 1 a 5 .

2. Administrar los posibles ítems: Distribución de ítems en muestra de personas (prueba piloto), seleccionadas al azar de una población con características similares a la de la Escala final.

3. Calcular la puntuación total: Suma del valor de cada ítem marcado.

4. Determinar el poder discriminativo: para la selección ítems de la escala final. Existe 2 métodos:

1) Consistencia Interna: Correlación de cada ítem en puntuación total reteniendo los coeficientes más altos. Este método también es conocido como Alpha de Cronbach.

2) Método de análisis de ítems: Examina habilidad de cada ítem, separa los muy favorables de los muy desfavorables, a esto se le 
llama Poder discriminativo (PD) de los ítems.

5. Selección de Ítems: Con los valores " $\mathrm{t}$ " para cada ítem se seleccionan los ítems que son significativos en un nivel de $\alpha$ determinado, o directamente aquellos con PD más grande.

6. Pruebas de confiabilidad: de escalas copilando más ítems para 2 escalas con un mínimo de 100 ítems, (Namakforoosh, 2000).

Por otra parte, Morales en 1981 y Arce en 1995 precisaron que la construcción de una escala tipo Likert supone las siguientes fases:

1. Preparación de los Ítems iniciales.

2. Administración de Ítems a una muestra de sujetos.

3. Asignación de los puntajes a los Ítems.

4. Asignación de puntuaciones a los sujetos.

5. Análisis y selección de los Ítems: Esto incluye los siguientes sistemas: Poder Discriminativo, Correlación Ítems-Test, Test de la Mediana, Test de probabilidad exacta de Fisher, Análisis Factorial, Contribución de cada Ítem a la Fiabilidad total de la Escala (Alpha de Cronbach).

6. Análisis de la fiabilidad de la escala: la fiabilidad de las escalas individuales de evaluación está en función directa del número de grados de la escala.

7. Preparación de la aplicación de la versión final de la escala, (Chica \& Castejón, 2006)

El establecimiento de la cantidad de Ítems pudiera estribar del fin que pretende el trabajo de investigación. Para esta investigación se considera analizar escalas de 1 a 5 y de 1 a 7 , y se seleccionará en la prueba piloto aquella que proporcione datos más fiables.

El análisis factorial pudiera ser un método útil para establecer la selección de ítems, el análisis factorial indica el grado en que las categorías se mezclan a lo largo del número limitado de dimensiones, (Hernández
Sampieri et al., 2014), es decir este proceso ayuda a determinar cómo afecta cada ítem en a toda la escala de medición.

Una ventaja de las Escalas Likert es la habilidad que tiene para probar la confiabilidad de cada escala por medio de la Alpha de Cronbach, el cual la interpretación de este estadístico está lejos de ser complicada (un valor alfa superior a 0,70 indica una escala fiable), (Spooren et al., 2007).

Estos métodos serán considerados para asegurar la efectividad del instrumento a utilizar.

\section{MÉTODO}

El presente estudio al ser parte de las ciencias sociales no contiene acciones experimentales y es una investigación de tipo descriptiva, realizada con la finalidad de fundamentar los aspectos teóricos para justificar la aplicación de Encuestas con Escala Likert, esto con la finalidad de precisar el grado de aplicación del Modelo de Control Interno COSO 2013 en las empresas públicas mexicanas, sin definición formal de este dato, es decir aquellas en las que no se puede precisar con información institucional publicada mencionado dato.

Se estableció como referencia el Modelo de Control Interno COSO 2013 considerando la popularidad que este tiene en las empresas públicas en Estados Unidos, precisando que la SEC y el PCAOB reconocieron como un marco de referencia adecuado sobre el control interno el emitido por COSO en 1992, (Buzo, 2014) y hoy en día la versión reciente de fue emitida en 2013 sigue prevaleciendo su renombre, dato que fue confirmado al analizar los informes anuales (formato 20-F) de 2019 de las 13 empresas públicas mexicanas que son públicas en los Mercados Financieros de Estados Unidos y la Bolsa Mexicana de Valores, donde se precisó la aplicación del Modelo de Control Interno COSO 2013 en estas (ver tabla 1).

Adicionalmente se fundamentaron las Disposiciones de Carácter General Aplicables a las Emisoras de Valores y otros participantes del Mercado de Valores en México las cuales no precisan el requerimiento de revelar el Modelo 
de Control Interno utilizado, como en el caso de Estados Unidos, en el que la SEC como órgano regulador de las compañías públicas en Estados Unidos, en sus reglas finales no exige el uso de un marco de Control Interno particular, como COSO 2013, considerando que existen otras normas de evaluación fuera de los Estados Unidos y que los marcos que no sean COSO pueden desarrollarse en el futuro dentro de dicho país, siempre que satisfagan la intención del estatuto sin disminuir los beneficios para los inversionistas, (US Securities and Exchange Commission, 2013).

Como resultado de esto se justificó la necesidad de confirmar el Modelo de Control Interno utilizado en nuestra población de estudio, estableciendo un instrumento de encuesta con Escala Likert para medir el grado de aplicación de COSO 2013, esto considerando la notoriedad y fundamentos que ha tenido este instrumento en diferentes ámbitos de la investigación, así como las facilidades y beneficios que este otorga.

\section{HIPOTESIS}

H1: Las encuestas con escala Likert precisan el grado de aplicación del Modelo de Control Interno COSO 2013 en las empresas públicas mexicanas, sin definición formal de este dato.

Ho: Las Encuestas con escala tipo Likert no precisan el grado de aplicación del Modelo de Control Interno COSO 2013 en las empresas públicas mexicanas, sin definición formal de este dato.

\section{RESULTADOS}

La carencia de datos medibles y regulaciones que requieran la revelación del Modelo de Control Interno aplicado por las empresas públicas mexicanas, precisan la necesidad de establecer estudios para: Identificar el Modelo de Control Interno utilizado, difundir los beneficios de cada Modelo de Control en México, evaluar la relevancia que dan las organizaciones a la cultura de Control y tomar como base esta para precisar aspectos como efectos en productividad, rentabilidad y la generación de valor en las organizaciones.

Considerando la problemática mencionada se podría justificar la aplicación de un instrumento de con Escala de Medición de Tipo Likert para calcular el grado aplicación del Modelo de Control Interno establecido en las empresas mexicanas sin definición de este dato.

El diseño y aplicación del instrumento de Encuesta deberá considerar los siguientes aspectos:

- Definir la encuesta bajo las bases de la Escala Likert.

- Los ítems de la encuesta deberán ser diseñados utilizando los fundamentos del Modelo de Control Interno COSO 2013.

- Realizar una prueba piloto con una muestra de personal con características similares a la población de estudio, es decir de las áreas de Control Interno y Auditoría.

- Con los datos obtenidos realizar las pruebas de confiabilidad correspondientes para precisar la idoneidad de la Encuesta con Escala Likert y discriminar los ítems que no favorezcan su fiabilidad.

- Definición del tamañ o de la muestra de estudio y establecer la estrategia para lanzar la encuesta final.

El producto final esperado al aplicar la encuesta será el diagnostico final del grado de aplicación del Modelo de Control Interno COSO 2013 en las empresas publicadas mexicanas sin definición de esta información y perfilar con esto estudios futuros para la medición de los efectos de dicho modelo en los aspectos que generan valor en las organizaciones en general, como el caso de Rentabilidad, Productividad, Eficiencia Operativa, entre otros.

\section{CONCLUSIONES}

Los aspectos fundamentados en este análisis buscan precisar la importancia que podría tener la aplicación de instrumentos de medición como la Escala de Tipo Likert para analizar las variables cualitativas que no cuentan con datos formales que precisen su estatus actual o nivel de aplicación y con esto pudiera detener el proceso de investigación o no fundamentar 
correctamente el mismo, por lo que a través de dicho análisis se pudiera determinar el grado de diligencia de la variable cualitativa en estudio y con esto analizar las características o resultados que conlleva su implementación, como es el caso de la rentabilidad o productividad generada por determinada variable de dicho tipo.

Es de relevancia precisar que la carencia de bases formales para identificar el Modelo de Control Interno aplicado en las empresas públicas mexicanas que no cuentan con la definición de este dato, es un área de oportunidad en la investigación científica, que al ser corroborada buscaría puntualizar la popularidad del Modelo de Control Interno COSO 2013 en México que es objeto de nuestro análisis, así como fomentar los efectos de su aplicación en las organizaciones y con esto las empresas y a nivel académico podrían tomar de bases científicas para su aplicación.

Se considera la definición de una Encuesta como instrumento de medición con escala de tipo Likert para buscar precisar de manera óptima el nivel de implementación del Modelo de Control Interno COSO 2013 en las empresas públicas mexicanas sin esclarecimiento formal de este dato, para el diseño de la encuesta se tomará como base los fundamentos teóricos de dicho modelo, así como las reglas a aplicar para la definición de una encuesta de la que se obtengan datos objetivos y confiables, que pudieran llevar a confirmar esta información, como son:

- Definición de una escala de evaluación adecuada.

- La aplicación de una prueba piloto con una población de características similares a la de estudio.

- Validación de la consistencia interna de la encuesta al aplicar la prueba de Alpha de Cronbach y el Análisis Factorial.

- Determinación de ítems finales a considerar en la Escala de Evaluación.

Bajo estas bases se fundamentaron los pasos para la utilización del instrumento de escalas de evaluación de tipo Likert para precisar el nivel de aplicación del Modelo de Control Interno COSO 2013 en las empresas públicas mexicanas en las que no se cuenta con definición formal de este dato o de su grado de implementación, así también pudiera ayudar a precisar la importancia que las organizaciones dan a los sistemas de control interno y bajo estas bases podría ser viable contar con información que permitiera medir los efectos en variables enfocadas a la generación de valor en las organizaciones como es el caso de la Rentabilidad, Eficiencia Operativa entre otros, para con ello buscar establecer bases científicas sobre los efectos de la aplicación de lo Modelo de Control Interno COSO 2013. 


\section{REFERENCIAS}

Arwinge, O. (2013). Internal control: A study of concept and themes. In Springer-Verlag Berlin Heidelberg (Ed.), Physica-Verlag a Springer Company. Springer-Verlag Berlin Heidelberg. https://doi.org/10.1007/978-3-7908-2151-2

Auditing and Assurance Standards Board. (2009). NIA 315 Identificación y valoración de los riesgos de incorrección material mediante el conocimiento de la entidad y de su entorno. 142. http://www.icac.meh.es/NIAS/NIA 315 p def.pdf

Buzo, J. (2014). Punto de vista. Año de Transición Al Nuevo COSO 2013., 1-6. https://studylib.es/doc/4935080/2014--año-de-transición-al-nuevo-coso-2013

Chica, A. A., \& Castejón, J. L. (2006). Elaboración, análisis e interpretación de encuestas, cuestionarios y escalas de opinión. In Universidad Alicante (Vol. 1). https://rua.ua.es/dspace/bitstream/10045/20331/1/Elaboración, análisis e interpretación.pdf

Commitee of Sponsoring Organizations of the Treadway Commission. (2013). Internal ControlIntegrated Framework (American Institute of Certified Public Accountants (ed.); Issue May, 2013).

González Lorenzo, M., Lameiras Fernández, M., \& Varela Lovelle, M. (1990). Escalamiento de magnitud en la satisfacción laboral: I. Validez de la ley de potencia. In Revista de psicología general y aplicada: Revista de la Federación Española de Asociaciones de Psicología (Vol. 43, Issue 3, pp. 411-417).

Hernández Campos, A., Martinez Perez, V. E., Luna, A., \& Hernande Flores, J. L. (2019). La auditoría de estados financieros para efectos fiscales como control interno para reducir las contingencias fiscales de las empresas (The financial audit for fiscal pourposes like fiscal internal control as a measure to contribute to the decreasing of. 16(31), 55-86.

Hernández Sampieri, R., Fernandez Collado, C., \& Baptista Lucio, P. (2014). Metodología de la Investigación (Vol. 6).

Hirt, R. (2015). Internal Auditor Middle East. Coso 'S Internal Control Framework, March, 16-19.

Namakforoosh, M. N. (2000). Metodología de la Investigación (Editorial Limusa (ed.)). https://books.google.com/books?id=ZEJ7-0hmvhwC\&lpg=PA283\&ots=i15wtTd0W\&dq=namakforoosh likert\&lr\&hl=es\&pg=PA243\#v=onepage \&q=likert\&f=false

Osinski, I. C., \& Bruno, A. S. (1998). Categorías de respuesta en escalas tipo likert. Psicothema, $10(3), 623-631$.

Secretaria de Gobernación. (2019). Disposiciones de carácter general aplicables a las Emisoras Valores y otros participantes del Mercado de Valores. Diario Oficial de La Federación.

Spooren, P., Mortelmans, D., \& Denekens, J. (2007). Student evaluation of teaching quality in higher education: Development of an instrument based on 10 Likert-scales. Assessment and Evaluation in Higher Education, 32(6), 667-679. https://doi.org/10.1080/02602930601117191

Sarbanes-Oxley Act, 44 (2002).

United States Securities and Exchange. (2021). FORM 20-F REGISTRATION STATEMENT PURSUANTTO SECTION $12(\mathrm{~b})$ OR $(\mathrm{g})$ OF THE SECURITIES EXCHANGE ACT OF 1934 OR ANNUAL REPORT PURSUANTTO SECTION 13 OR 15 ( $d$ ) OF THE SECURITIES EXCHANGE For the fiscal year ended 1852. 
US Securities and Exchange Commission. (2013). Final Rule: Management's Report on Internal Control Over Financial Reporting and Certification of Disclosure in Exchange Act Periodic Reports; Rel. No. 33-8238. https://www.sec.gov/rules/final/33-8238.htm 